\title{
Critical defect size assessment in pipelines on a nuclear power plant
}

\author{
Galya Dimova, ${ }^{1, *}$, Vladimir Yurukov $^{2}$, Kiril Djivdjanov ${ }^{3}$ \\ ${ }^{1}$ Kozloduy NPP EAD, ICC-DCC, 3321 Kozloduy, Bulgaria \\ ${ }^{2}$ ATP - AtomToploProekt" LTD, Sofia, Bulgaria \\ ${ }^{3}$ SENSATA, Sofia, Bulgaria
}

\begin{abstract}
In many energy industry structures, pipeline systems are subject to the impact of mechanical forces, moments of forces and fluid flows of high pressure and temperature. These load factors cause defects in the pipeline metal. As the years of operation increase, defects may occur and grow, which may lead to the destruction of pipeline walls. Special measures have been planned and implemented to ensure the safe operation of high-energy facilities. This study focused on pipelines and nozzles of nuclear power plant equipment with bimetal welded joints on which the size of critical defects was assessed. The base of assessment covers material properties, temperature and stress fields, fracture mechanics calculations. This study involves developing of finite element models and implementing simulations on them in order to obtain temperature fields and determine the stress-strain state of the component.
\end{abstract}

\section{Introduction}

In many energy industry structures, pipeline systems are subject to the impact of mechanical forces, moments of forces and fluid flows of high pressure $17 \div 18 \mathrm{MPa}$ and temperature $330 \div 350{ }^{\circ} \mathrm{C}$ After years of operation under such conditions discontinuities (defects) can occur in the metal walls of the pipelines [1], as well production defects (from steel's melt) can appear. It is known that defects can remain stable without development, or, conversely, defects can grow further. Critical defect's size is a condition which produces a rapid and unexpected destruction of a component (fast fracture). The presence and size of defects is detected through nondestructive testing (NDT) methods, while strength analyses taking into account a load factors can forecast their locations with high probability - the so called critical areas in the facilities.

This paper considers a pipe with both cladding and thermal shirt (shield, jacket) made of austenitic stainless steel - a different steel type from that of the pipe itself. In some cases, the thermal shirt can be welded to the pipe with bimetallic welded joints. The two types of welded metals have different thermal characteristics. Under the impact of high temperature and pressure values of the fluid in the pipeline systems typical for the RPV (reactor pressure vessel), and following multiple load cycles, stress zones with higher strains can form in the boundary of welded joints. The difference in thermal properties of steels in bimetallic welds suggests that they will expand and contract at different rates during the plant modes of warming and cooling. Occurrence of discontinuities in the critical zones is expected as well as their further growth under the influence of strain values throughout the 
different operation conditions NOC (Normal Operating Conditions), DNOC (Deviation of Normal Operating Conditions) thus turning into defects of metal [2]. To ensure safe operation of NPP pipeline systems with bimetallic joints, it is important to specify/predict the place and the growth of discontinuities. Prediction of the defects behaviour is essential. Such information will enable the prioritised examination of these critical zones in full scope and using testing techniques ensuring high data resolution. The process of studying discontinuities and justifying their behaviour provides a modern approach to safe operation of the facilities with relatively low investments.

The purpose of this paper is to present the approach for detecting of critical zones in bimetallic pipe nozzles, where it is justified to presume that defects will appear, and assess the defects' critical dimensions. This study used a really existing object - nozzles of the reactor pressure vessel emergency core cooling systems. A 3D FEM (Finite Element model) model of the object of study was prepared in which commercial software product was used to made the geometry, the finite elements mesh and then to imitate the load factors (pressure, temperature, forces\& moments) in the different operating modes of a nuclear power plant. The strength analyses identified the critical zones where series of discontinuities (defects) are postulated. These can be different types of defects, such as planar ones of the crack type, axially oriented as well as circumferentially - (postulated as) semi elliptical, located on the interface surface (i.e. sub clad ones) or elliptical internal ones (i.e. in the pipe wall, far from the cladding and outer surface). Growth of defects under the operating environment impact was imitated using software. A limiting condition for the calculations is that these defects should not lead to a fast fracture of the nozzle. Through solving the inverse problem, or reverse iteration, the maximum size defect is determined that, on the one hand, can be detectable via ultrasonic nondestructive testing and, on the other, the defect will not reach its critical size throughout the planned operational lifetime of the equipment.

The study is expected to assist in the development of the processes of repair, maintenance, and monitoring of pipeline systems as well as the projects for their long-term operation once their design lifetime has been reached. The study is also intended to support the systems qualification processes of nondestructive testing, and to ensure risk-informed control.

\section{Methodology}

\subsection{Calculations of strains-stresses fields}

Unstable condition of reactor unit equipment most often occurs under DNOC and EC Postulated Accidents (Emergency Conditions). On the other hand, the defects develop due to the cycle loads of the nozzle, affected by different mechanical and thermal influences typical for the NOC, DNOC, and HT modes (HT - Hydrotest or Pressure Test). The number of the actual repeats of the operations transient modes was taken from the I\&C ( I\&C - Instrumentation and Control System for technological data collection measured by sensors) and MDS System measurements (MDS - Monitoring and Diagnostic system for collection of supplementary data (temperature of the metal, stress and strain, etc.) The calculations of stress and strain fields cover all the operation modes of the nuclear power plant [2]. The calculations have considered specific load factors typical for Nuclear Power Plant, [3] a) inside / outside pressure in the pipeline system; b) pipeline weight; c) fluid weight; d) residual stress after welding; e) seismic loads; f) load originating from supports; g) dynamic forces of fluid pulsations, vibrations; h) stresses generated by temperature differences and differences between the materials thermal expansion coefficient (base 
metal, weld metal); j) stresses due to pressure and thermal fluctuations. To complete the structural analyses a $3 D$ finite elements model (FEM) of the pipeline was created, using CAE module pack NISA (originally developed by EMRC co, now Crane co), [3].

The first stage of the structural calculations covers assessment of thermal transients and included simulations of the transients' heat transfer (taken into account processes of convection and conductivity) at different operational transient modes. The pipeline temperature distributions in the model of the tube and nozzle for the different moments of the analyzed temperature transients were produced by FEM simulations using NISA's the HEAT III module.

The second stage of the study considers the mechanical loads added to the thermal ones. Strain-stress conditions (SSC) for every characteristic situation of transients is defined by FEM simulations using module NISA II. The result represents the distribution of total stress due to both thermal and mechanical loads.

After the structural stress calculations, the pipeline nozzle sections with the greatest equivalent stresses were defined.

\subsection{Size assessment of the critical defects}

Following the structural calculations, in the critical zones thus identified, the presence of a family/series of defects are postulated, supposing every defect's semi-axis have a size of $a_{0}=a+\Delta_{a}$, Fig. 1 .

$a$ - depth (minor semi-axis) of a schematized (semi-)elliptical defect;

$\Delta_{a}$ - an uncertainty value followed by ultrasonic testing UT procedure

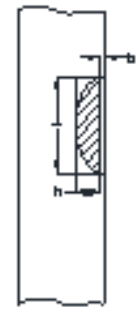

a.

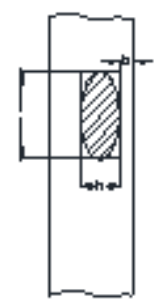

b.

Fig. 1. Defect schematization, $h=a_{0}$ - semielliptical, $h=2 a_{0}$ - elliptical, for both types $-l=2 c ; h$ - depth of a schematized (semi-) elliptical defect, $l$ - length of a schematized (semi-) elliptical defect; $b$ - size of a ligament. If the ligament, $\mathrm{b}$, between the defect and outer surface is smaller than $a_{0} / 9$, then the defect is considered a (conditional) surface one
(a) Semielliptical conditionally surface defect
(b) Elliptical inner defect (embedded crack).

Under the different operating conditions of the component, the defect will be expected to develop by growing (however, it is also possible that the defect will not grow within the operational lifetime limits). The growth of the defect $d a$ can be detected by nondestructive testing of the pipeline zone, with an uncertainty value of $\Delta_{a}$. In reality, on NPPs in operation, non-destructive tests on components are not performed continuously, but at specified time intervals. Considering the intervals between pipeline NDTs and the increment value $d a$ over one interval, the defect will reach a size of: 


$$
a_{1}=a_{0}+d a ; \text { or } a_{1}=a+\Delta_{a}+d a
$$

Repeating this step, after $n$ NDT intervals the defect will reach the size of:

$$
a_{n}=a_{n-1}+\Delta_{a_{n-1}}+d a_{n-1}
$$

This step is reiterated until the end of the pipeline system operating lifetime (limiting condition), while the defect should not reach its critical size during this limiting timeframe. The target is to define that value of $a_{n}$, for which the defect will not reach its critical size $a_{\text {crit }}$, or $a_{n} \leq\lfloor a\rfloor$

$[a]$ - critical size of a schematized (semi-) elliptical defect

The growth rate of the defect $d a$ is defined according to the Methodology [1].

The defect growth of a component subjected to cyclic loads is calculated by the Paris model $[1,2]$.

$$
\frac{d a}{d N}=C_{0} \cdot\left(\frac{\Delta K}{\sqrt{1-R}}\right)^{m}
$$

$N$ - number of load cycles; $\Delta K=K_{\max }-K_{\min }$ - range of stress intensity factor

$C_{0}, m$ - material properties; $R$ - loading cycle asymmetry coefficient

The limit condition of a pipeline with a defect is determined as follows [3]:

- for ferritic components (SIF criterion or fracture toughness criterion):

$$
K_{I} \leq\left[K_{I}\right]
$$

- for ferritic and austenitic components (limit load failure (LLF) criterion)

$$
R_{F}{ }^{T}=1.2 \cdot R_{P_{0.2}}{ }^{T}
$$

$K_{I}$ - stress intensity factor, $M P a \cdot \sqrt{m}$

$\left[K_{I}\right]$ - allowable value of stress intensity factor, after applying all safety factors

$R_{P 0.2}$ - yield strength, $\mathrm{MPa}$;

A dedicated software code developed specially for the current work was used to determine the nozzle critical defect size.

\section{Input data}

The input data include drawings of the Dn300 nozzle ( $D n$ - diameter nominal of the tube) and actual geometric size values; material data and thermo-hydraulic loads under different plant operating conditions. The results provided apply to the Dn300 nozzles on the hydraulic accumulators (HA) of the upper and lower plenum chambers of the reactor pressure vessel (RPV) with a thermal sleeve of the passive system for ECC. The function of the Emergency Core Cooling System (ECCS); is to quickly inject boric acid solution in the RPV to cool down the core, and flood it in case of a loss of coolant accident (LOCA) when 
the pressure $\left(\mathrm{P}_{1}\right)$ in the primary circuit drops below $5.9 \mathrm{MPa}$. The ECCS passive part comprises tanks (HA), Dn300 connecting pipelines between the HA and the RPV, and valves on the pipelines. The four Dn300 nozzles between the ECCS passive part and the RPV come in couples - two of which lie on the beltline diametrically opposite of the Dn 850 of the lower plenum chamber, and another two nozzles located on the beltline diametrically opposite of the Dn 850 of the upper mixing chamber. All the four Dn 300 nozzles have similar geometry. Each of them has a thermal mantle (jacket) from stainless $\mathrm{Cr}-\mathrm{Ni}$ austenitic steel welded through bimetal Y-type welded joints.

Stress calculations are performed taking into account the internal pressure, dead weight and temperature gradients. Residual stresses in welding joints and in cladding are calculated for all operating conditions. The loads considerations in NOC are as follow [3]:1) Planned heating from cold condition to normal work condition at a rate of $20^{0} C / h ; 2$ ) Spurious actuation of the RPV emergency protection; 3) Planned cooling from normal work condition to cold condition at a rate of $30^{\circ} \mathrm{C} / \mathrm{h}$; 4) Weight of the nozzle; 5) Total seismic load; 6) Heat transfer from pipeline system.

The loads considerations in DNOC are: 1) Shutdown of turbine valves; 2) Loss (total) of coolant; 3) Stopping of water feed to steam-generator; 4) Breaking of a steam-generator heat exchange tube; 5) Water injection (temperature $60 \div 70^{0} C$ ) in pressuriser; 6) RPV cooling at a rate of $60^{\circ} \mathrm{C} / \mathrm{h}$.

\section{Results and discussion}

The loads model and the strength calculations used 15 transients and conditions typical for ECCS for the Dn300 nozzle. Two emergency conditions regarded as representative for the defects growth were considered: a) in case $P_{I}<5.9 \mathrm{MPa}$, and b) in case the passive component of ECCS should be actuated. The trend-curves of pressure $P_{I}(t)$ and temperature $T(t)$ are shown on Fig. 2.

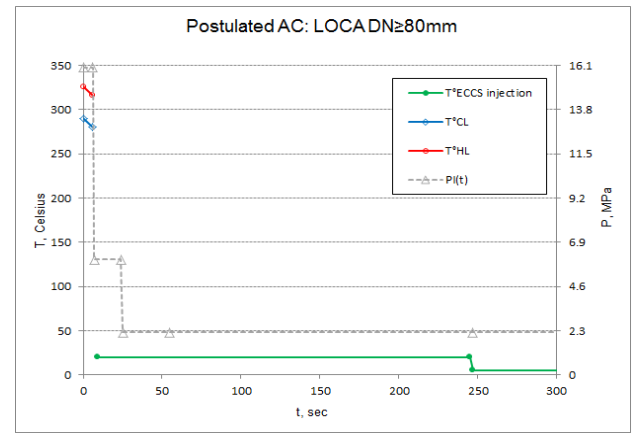

Fig. 2a. The trend-curves of pressure and temperature in LOCA: rupture of pipeline values of the coolant temperature, coming out from the RPV; values of the coolant temperature, coming in the $\mathrm{RPV}$; injection: boric acid injected in the RPV (CL/HL - cold/hot legs) 


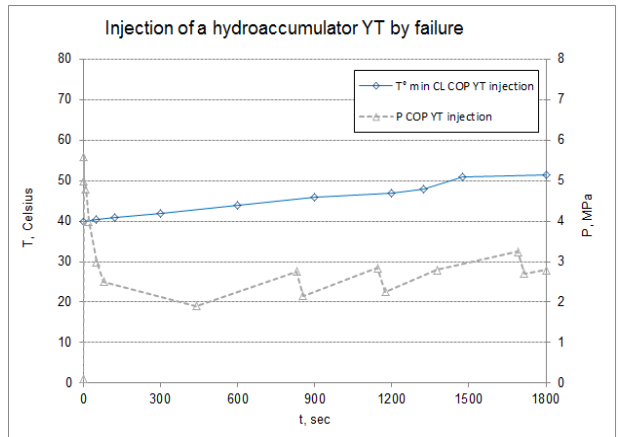

Fig. 2b. The trend-curves of temperature in EC: boric acid is wrongly injected into one of the accumulators YT. CL COP YT injection: values of the temperature of the coolant in the RPV (lower plenum chamber).

FEM of nozzle Dn300 is presented on Fig. 3 .

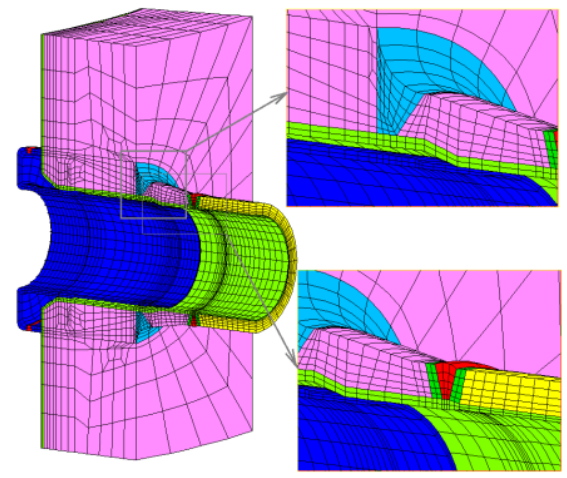

Fig. 3. FEM of nozzle Dn300 . Ferritic welded joint (up) and bimetal welded joint (down)

The heat transfer transients of Dn300 nozzle FEM are shown on Fig. 4 . The initial and the boundary conditions for the thermal conductivity and structural calculations are illustrated. The process for determining the strength-stress condition of the Dn300 nozzle is demonstrated.

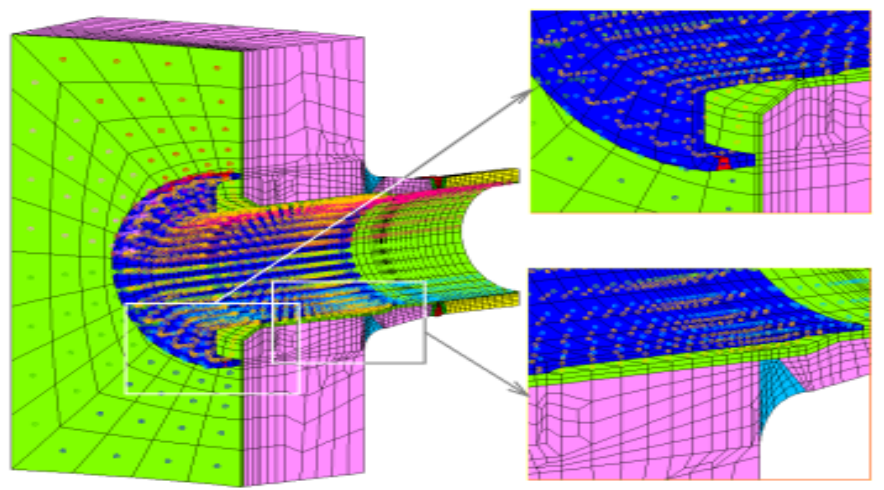

Fig. 4. Dn300 nozzle FEM - Boundary conditions applied for Heat transfer simulations (up) and for obtaining stress fields (down). 
The stress zones with maximum stresses are received by large selection of typical transients. The assessment of stress zones was based on the analysis of the distribution of the axial $(S z z)$ and circular ( $S y y)$ stresses due to thermal loads under LOCA with $D n \geq 80 \mathrm{~mm}$ rupture. Some typical transients were selected and the areas (cross-sections) with maximum stresses were identified, Fig.5.
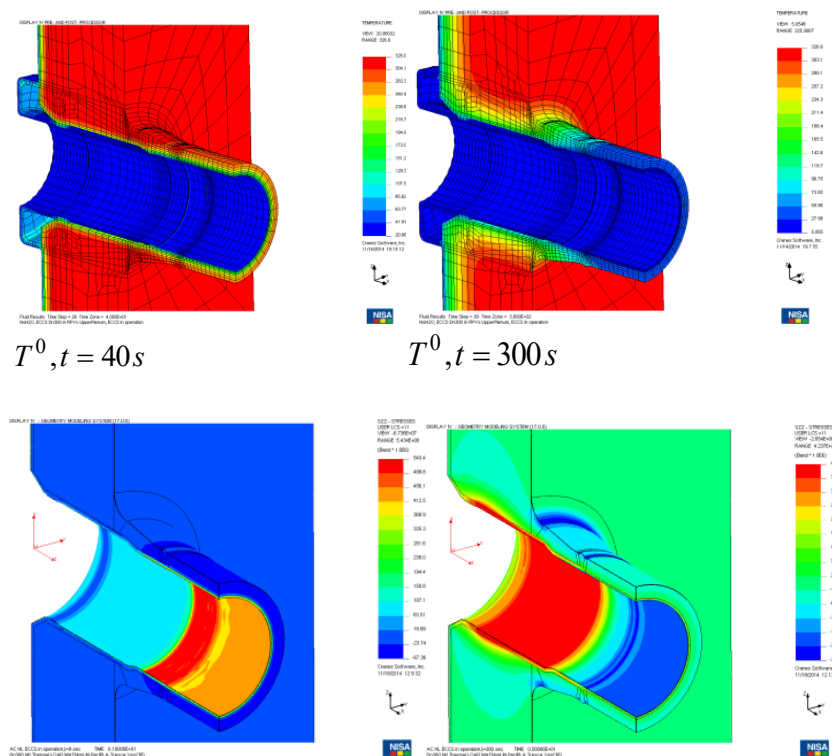

$S z z, t=9 s$

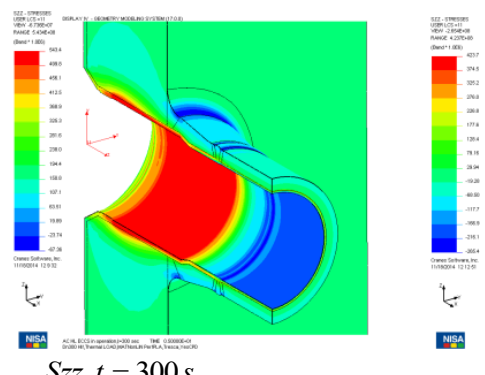

Fig. 5. Areas of ECCS Dn300 nozzle subjected to transient at LOCA Dn $\geq 80 \mathrm{~mm}$. Distribution of the temperature $\mathrm{T}$ at $t=40 \mathrm{~s}$ and $t=300 \mathrm{~s}$ (up). Distributions of the axial ( $\mathrm{Szz}$ ) stresses due to thermal loads at $t=9 \mathrm{~s}$ and $t=300 \mathrm{~s}$ (down).

The cross-sections for which calculations provided according to [1] are shown on Fig.6. The defects position was postulated at cross-sections $1 \div 5$ and their size growth was calculated according to the Methodology [1]. The defects behaviour was assessed in terms of "worst-case scenario" for the defect's location and growth. The criterion for an admissible defect is $h / l=0.15,[3]$.
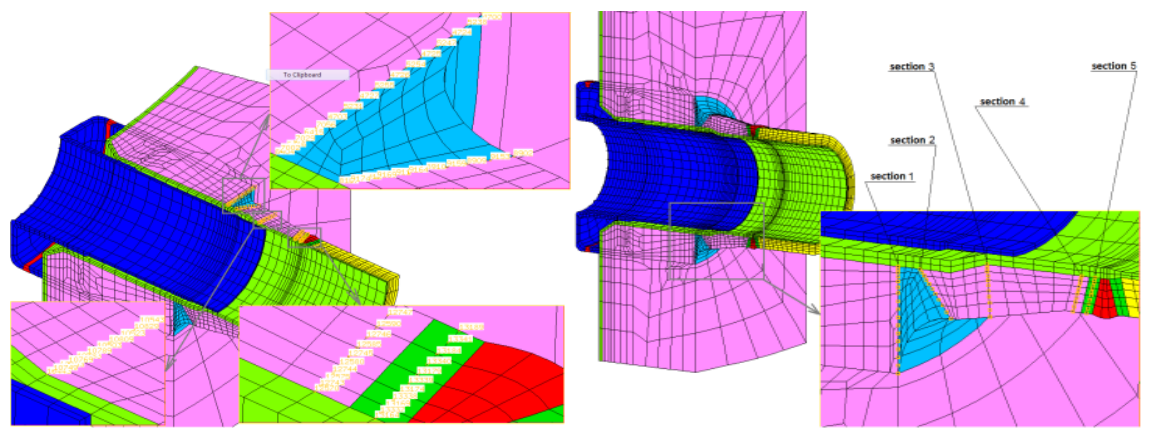

Fig. 6. Cross-sections for which calculations are provided according to [1]. Cross-section areas $1 \div 5$ with maximum axial ( $S z z$ ) stress due to thermal loads. 
The results demonstrate two types of "worst-case" defects: an axial defect, situated very closely to the ferritic welded joint (cross-section 2) and a circle defect, situated closely to the bimetal welded joint (cross-section 4).

Based on the calculations, the development for the postulated axial surface defect reaches values of, respectively $2.0 \mathrm{~mm}$ for the period of 4years and $9.4 \mathrm{~mm}$ for the period of 30 years. The development for the postulated circle surface defect reaches respectively $0.8 \mathrm{~mm}$ for the period of 4years and $4.4 \mathrm{~mm}$ for the period of 30 years.

Maximum growth for the surface defect is received for an axial defect, in ferritic crosssection 1 - defect reaches respectively $6.4 \mathrm{~mm}$ for the period of 4 years and $26.1 \mathrm{~mm}$ for the period of 30 years. The results for defects' growth for a period of 4years are presented in Table 1.

Table 1. The sizes of critical defect growth after 4 years in nozzle Dn300, with ratio of semi-axis 0.3 and 0.7

\begin{tabular}{|c|c|c|c|c|c|c|c|c|c|}
\hline \multicolumn{10}{|c|}{ For conditional surface defect, $[\mathrm{mm}]$} \\
\hline \multicolumn{5}{|c|}{0.3} & \multicolumn{5}{|c|}{0.7} \\
\hline$a$ & $2 c$ & $h / l$ & $h$ & $l$ & $a$ & $2 c$ & $h / l$ & $h$ & $l$ \\
\hline 6.0 & 40.4 & 0.15 & 6.0 & 40 & 7.7 & 22.0 & 0.35 & 7.7 & 22.0 \\
\hline \multicolumn{10}{|c|}{ For embedded defect, $[\mathrm{mm}]$} \\
\hline 3.65 & 24.3 & 0.3 & 7.3 & 24.3 & 4.7 & 13.3 & 0.7 & 9.4 & 13.4 \\
\hline
\end{tabular}

\section{Conclusions}

The research presents an approach for definition of critical zones in bimetallic welded joints on pipeline nozzles for which there is evidence to assume potential occurrence of defects after many years of operation. Presence of defects is postulated for these zones, and the critical defect sizes are calculated. The approach for the defects' critical size assessment is underpinned by regulatory requirements. Special assessments of strain-stress condition are made.

Based on the assessment of thermo-hydraulic loads, it can be possible to identify comparatively precisely the spots for non-destructive testing. The study is intended to assist in the development of the process of non-destructive testing of NPP equipment, as well as for technical diagnostics of the components.

\section{References}

1. VNiiAEC, Methodology for definition of the allowable sizes of the defects in the metal of the equipment and pipelines in NPP, M-02-91 (Russia, 1991, rev. from 1997),

2. IAEA, Unified Procedure for Lifetime Assessment of Components and Piping in WWER NPPS, “VERLIFE Procedure" (Austria, 2012)

3. Code of Strenght Calculation of Equipment and Pipelines of Nuclear Power Plant, PNAE G-7-002-86, (Russia, Energoatomizdat, 1986) 